\title{
Escala de Crenças sobre Amor Romântico: Indicadores de Validade e Precisão ${ }^{1}$
}

\author{
Alexsandro Luiz de Andrade ${ }^{2}$ \\ Agnaldo Garcia \\ Universidade Federal do Espírito Santo
}

\begin{abstract}
RESUMO - Crenças individuais comumente interagem na dinâmica de envolvimento entre os membros de um relacionamento romântico, influenciando, entre outros aspectos, os padrões de percepção, construção de expectativas e estratégias de relacionamento. Esta pesquisa teve por objetivo construir, validar e confirmar a estrutura dimensional de uma medida para avaliação de crenças sobre o amor em uma população brasileira. Participaram da pesquisa 1530 pessoas, sendo 660 do sexo masculino e 870 do sexo feminino subdivididas em duas subamostras. Os resultados apontaram um instrumento psicométrico com parâmetros de precisão e confiabilidade adequados e confirmaram uma estrutura dimensional de seis fatores de estilos de amor na população brasileira: eros, agape, ludus, storge, mania e pragma.
\end{abstract}

Palavras-chaves: relacionamentos românticos, estilos de amor, medida psicológica

\section{The Scale of Beliefs about Romantic Love: Indicators of Validity and Internal Consistency}

\begin{abstract}
Aspects of beliefs commonly interact in the dynamics of romantic relationships, thus influencing aspects of the perception, expectations and relationship strategies, among others. This research aimed to construct and validate a measure assessing beliefs about love in a Brazilian sample and verify its dimensional structure. A total of 1,530 individuals participated in this study, 660 of these being males and 870 females: The sample was divided into two sub-samples. The results showed a psychometric instrument with adequate parameters of precision and a confirmed structure of six-dimensional factors styles of love in a Brazilian sample: eros, agape, ludus, storge, mania and pragma.
\end{abstract}

Keywords: romantic relationships, love styles, psychological measurement

Relacionar-se romanticamente é parte integrante da vida de quase todas as pessoas pertencentes a praticamente todas as culturas mundiais. Talvez por essa importância e centralidade na vida das mesmas, há aproximadamente três décadas o estudo dos relacionamentos românticos vem convertendo-se em uma área de intensa demanda e inquietude por parte dos psicólogos do meio científico (Alferes, 1996; Sánchez Aragón, 2005).

Para compreender a natureza geral do amor, um grande número de teorias e pesquisas relativas ao assunto são propostas. Entre as mais importantes, segundo meta-análise de Masuda (2003), estão: a) a teoria dualista do amor (Berscheid $\&$ Walster, 1969), a qual distingue duas maneiras de interação romântica: o amor paixão e o amor companheiro; b) a concepção triangular do amor de Sternberg (1986), a qual propõe uma estrutura com três componentes essenciais para entendimento dos relacionamentos românticos: a intimidade, a paixão e o compromisso; c) a abordagem de caráter evolutivo/etológico, baseada no estudo de Hazan e Shaver (1987), a qual explicita a relação entre os padrões de apego na vida infantil e suas repercussões no desenvolvimento das relações românticas na vida adulta; e d) a teoria de John Alan

1 Apoio: CAPES, CNPq e Fapes/ES

2 Endereço para correspondência: Programa de Pós-Graduação em Psicologia, Av. Fernando Ferrari, n 514, Campus Universitário de Goiabeiras/UFES-CEMUNI VI, Vitória-ES, CEP 29075-910. E-mail: alexsandro.deandrade@yahoo.com
Lee (Lee, 1977) sobre os estilo de amor, concepção foco da medida desenvolvida neste estudo.

A concepção tipológica do amor - The Colors of Love (Hendrick \& Hendrick, 1986, 2006; Hendrick, Hendrick, \& Adler, 1988; Hendrick, Hendrick, \& Dicke, 1998), representa os estilos de amor compreendidos como aspectos do domínio cognitivo ligados ao sistema de crenças e atitudes do indivíduo sobre as situações românticas de sua vida. Estes incluem no seu núcleo elementos relacionados a variáveis emocionais e traços de personalidade. Segundo a taxonomia dos estilos de amor, o seu conjunto forma um círculo fechado, no qual se enquadram três estilos primários de amor (eros, ludus e storge) e três estilos secundários, formados a partir da combinação dos primários (mania, pragma e Agape).

Os seis construtos de estilos de amor segundo Lee (1977) são: a) Eros, padrão românticos caracterizado por valorização de atributos de ordem física e sexual na interação conjugal; b) Ludus, um tipo de amor manipulativo, marcado por jogos entre parceiros. O indivíduo que possui um estilo de amor predominante em Ludus interage dentro do relacionamento de maneira mais descomprometida, faz da interação conjugal um "jogo"; c) Storge, caracterizado por um estilo de relacionamento mais amigável e companheiro. Maior tendência a esta dimensão permite que as pessoas atribuam a seus parceiros fortes representações de amizade; d) Pragma, o indivíduo característico deste estilo opera num nível mais racional do que emocional, possui um estilo mais lógico e calculista nos seus relacionamentos; e) Mania, é o estilo de amor 
Tabela 1. Distribuição amostral dos participantes do Estudo III

\begin{tabular}{lllll}
\hline Variável & & Exploratória $(\mathrm{N})$ & Confirmatória $(\mathrm{N})$ & Total $(\mathrm{N})$ \\
\hline \multirow{2}{*}{ Sexo } & Masculino & $312(40,5 \%)$ & $348(45,8 \%)$ & $660(43,1 \%)$ \\
& Feminino & $458(59,5 \%)$ & $412(54,2 \%)$ & $870(56,9 \%)$ \\
\hline \multirow{2}{*}{ Escolaridade } & Fundamental & $14(1,8 \%)$ & $4(0,5 \%)$ & $18(1,2 \%)$ \\
& Médio & $120(15,6 \%)$ & $111(14,6 \%)$ & $231(15,1 \%)$ \\
& Superior & $636(82,6)$ & $645(84,9 \%)$ & $1281(83,7 \%)$ \\
\hline \multirow{2}{*}{$\begin{array}{l}\text { Vivenciava relacionamento } \\
\text { no momento do estudo }\end{array}$} & Sim & $544(71,3)$ & $487(64,7 \%)$ & $1031(68 \%)^{*}$ \\
\hline
\end{tabular}

*Valores com presença de casos omissos.

mais intenso. As pessoas que carregam elevadas pontuações nestas dimensões vivem a experiência romântica de maneira muito intensa, possessiva e irreal. É um amor considerado imaturo e não saudável; e f) Agape: que é o estilo amoroso altruístico, muito raro de ser manifestado isoladamente, é um amor caracterizado pela doação excessiva do indivíduo ao seu companheiro(a).

A metodologia criada para avaliar esses estilos foi adaptada no formato de escalas do tipo Likert de avaliação por Hendrick e Hendrick (1986). A versão original da escala é constituída por um total de 42 itens, sendo alocados sete itens por dimensão. Uma versão reduzida do instrumento, com indicadores de confiabilidades razoáveis foi validada por Hendrick, Hendrick e Dicke (1998). Estudos em diversas culturas encontraram resultados semelhantes aos dos autores da versão original. Em pesquisa transcultural, Neto et al. (2000) utilizaram uma versão da medida em diversos países (Portugal, Angola, Moçambique, Cabo Verde, Brasil, França, Suíça) encontrando resultados favoráveis na confirmação da estrutura dimensional da medida. Kanemasa, Taniguchi e Daibo (2004) realizaram um estudo com resultados semelhantes no Japão, Sánchez Aragón (2005) no México, Ferrer Pérez, Bosch Fiol, Navarro Guzmán, Ramis Palmer e García Buades (2008) na Espanha, De Andrade e Garcia (2009) e Cassep-Borges (2010) apresentaram versões preliminares do instrumento no Brasil nos últimos anos.

Apesar de já passados mais de 30 anos do primeiro trabalho de Lee sobre os perfis amorosos, o tema ainda é bastante pesquisado. White, Hendrick e Hendrick (2004) encontraram diversas associações em um recente estudo entre variáveis de personalidade, mensuradas pelo modelo dos cinco fatores e os seis estilos de amor propostos, concluindo que traços de neuroticismo apresentaram correlações positivas com estilo mania, bem como estilo eros associando-se positivamente com o traço extroversão. Em Colins e Read (1990), foi estudada, também de forma escalar, a relação dos estilos de amor e apego, destacando-se o resultado de correlação positiva entre apego do tipo ansioso e um estilo romântico do tipo mania. Em Contreras, Hendrick e Hendrick (1996) foram encontrados indícios significativos entre os estilos de amor mais apaixonados, como mania e eros, e um grau mais elevado na satisfação com o relacionamento de casal. Já em Vohs, Finkenauer e Baumeister (2010) são observadas relações positivas entre padrões de crença do tipo pragma e Agape com elementos de autocontrole, pessoas com escores altos nos dois estilos são mais sérias e comprometidas em relacionamentos românticos, estilos considerados preditivos da qualidade global em relações românticas.

O presente estudo possui por objetivos a apresentação dos indicadores de confiabilidade, validade fatorial exploratória e confirmatória da versão reformulada da Escala de Crenças sobre Amor Romântico para o contexto brasileiro, bem como a relação das diferentes dimensões da medida resultante com aspectos de qualidade no relacionamento, término ou não da relação e sexo.

\section{Método}

\section{Participantes}

A amostra deste estudo possui um caráter de conveniência e contou com um total de 1530 pessoas. Os participantes foram divididos em dois subgrupos amostrais, a primeira parte da amostra foi relacionada às análises exploratórias da medida psicológica utilizada, e a segunda voltada para verificação confirmatória da estrutura dimensional do instrumento.

Os dados gerais relacionados à distribuição da amostra são apresentados na Tabela 1.

Com objetivo de estruturar uma medida capaz de cobrir características demográficas de diferentes regiões do território brasileiro, a coleta de dados expandiu-se para quatro das cinco regiões do território nacional, buscando desta forma, mesmo que num nível parcial, levantar comportamentos representantes de pessoas de cada território.

Os dados demográficos relativos à distribuição geográfica dos participantes estão descritos na Tabela 2.

A primeira parte do estudo contou com 770 pessoas de ambos os sexos, sendo 312 (40,5\%) do sexo masculino, e $458(59,5 \%)$ do sexo feminino. A média de idade dos participantes foi de 25,5 anos ( $\mathrm{DP}=8,7$ anos). Dos participantes desta parte do estudo, 544 (70,6 \%) afirmaram estar vivendo um relacionamento romântico no momento da consulta, 
Tabela 2. Distribuição por região dos participantes

\begin{tabular}{lccc}
\hline Estado & Exploratória (N) & Confirmatória (N) & Total \\
\hline Espírito Santo - ES & 324 & 294 & 618 \\
Santa Catarina - SC & 286 & 316 & 602 \\
Paraíba - PB & 101 & 89 & 190 \\
Pernambuco PE & 23 & 24 & 47 \\
Amazonas - AM & 36 & 37 & 73 \\
\hline Total & 770 & 760 & 1530 \\
\hline
\end{tabular}

o restante não. A média de tempo dos relacionamentos da amostra foi de 53,5 meses ( $\mathrm{DP}=79,8$ meses). A amostra para o estudo confirmatório de validade da medida contou com 760 participantes: destes, $348(45,8 \%)$ eram do sexo masculino, e $412(54,2 \%)$ do sexo feminino. Dos participantes desta parte do estudo, $487(64,1 \%)$ declaram estar envolvido em um relacionamento romântico, com duração média de 41,97 meses ( $\mathrm{DP}=67,2$ meses).

\section{Instrumento}

O instrumento desenvolvido neste estudo é uma versão reformulada da escala LAS-Bra (De Andrade \& Garcia, 2009). A versão original do instrumento brasileiro foi validada em uma amostra de internautas, obtendo coeficientes de confiabilidade de 0,55 a 0,81. A medida foi construída a partir de procedimentos de tradução, validade de face e fatorial, a partir da escala de Hendrick e Hendrick (1986). A nova versão brasileira da medida recebeu o acréscimo e alteração de alguns itens que tiveram baixa carga fatorial na versão inicial. No total, 70 itens foram estruturados e dispostos para avaliação via uso de escalas de concordância de cinco pontos (discordo fortemente à concordo fortemente). A medida foi reestrutura tomando como base os seis fatores da teoria das cores de amor de Lee (1977): Eros, Storge, Ludus, Mania, Pragma e Agape.

Juntamente com o instrumento proposto, um questionário sóciodemográfico com questões de caracterização dos participantes, bem como perguntas sobre variáveis gerais do relacionamento, foi colocado (idade, sexo, estar num relacionamento ou ter terminado um relacionamento, etc). Com intuito de verificar aspectos convergentes e divergentes com outros fenômenos, no questionário geral acrescentou-se a Escala Multidimensional para Avaliação de Qualidade em Relacionamentos Românticos (Aquarela-R). Tal instrumento foi criado por De Andrade e Garcia (2012) e avalia diretamente cinco dimensões cognitivas, afetivas e comportamentais de qualidade dos relacionamentos românticos: a) comprometimento; b) intimidade; c) amor; d) relacionamento sexual; e e) comunicação, sendo que no presente estudo a dimensão amor foi excluída por apresentar número de respostas em branco superior a $5 \%$. A precisão do Aquarela-R a partir dos índices de confiabilidade alfa de Cronbach foram superiores a 0,90 em todos os fatores.

\section{Análise de dados}

Os dados da pesquisa foram analisados com auxílio do programa SPSS (Statistical Package for the Social Sciences) versão 13.0, e Amos (Analysis of Moment Structures) versão 7.0. Inicialmente, realizaram-se cálculos de estatística descritiva com todos os itens da escala. Primeiramente foi verificada a estrutura dimensional do instrumento por meio da análise fatorial exploratória e cálculos dos índices de confiabilidade alfa de Cronbach para os itens das subescalas resultantes. Com a segunda amostra do estudo foram realizados procedimentos fatoriais confirmatórios (CFA) e também o cálculo dos referidos coeficientes de confiabilidade das escalas resultantes.

Para os testes de ajuste dos modelos propostos na CFA, o método de estimação adotado foi o ML (Maximum Likelihood). Foram analisados os seguintes índices de acordo com as sugestões de Byrne (2010) e Hair, Anderson, Tatham \& Black (2005): a) $\chi 2$ (qui-quadrado), indicador que avalia a probabilidade do modelo selecionado se ajustar ao dados da matriz; b) $\chi^{2} / g .1$. - indicador de ajustamento, recomendam-se valores entre 2 e 3 , admitindo-se até 5; c) CFI (Comparative Fit Index ou índice de ajuste comparativo), que é um indicador comparativo referente ao ajuste dos modelos, seus valores variam de 0 a 1 , quanto mais próximos de 1, melhor ajuste, sendo os valores superiores a 0,90 adotados para aceitação do modelo; d) RMSEA (Root Mean Square Error of Aproximation ou Raíz quadrada media do erro de aproximação), que é um indicador de resíduos, sendo recomendados valores situados entre 0,05 a 0,08 , com intervalo de confiança de $90 \%$, aceitando-se valores de até 0,10 , este índice é mais indicado para estratégias confirmatórias de grandes amostras; e) GFI (Goodness-of-fit Index ou índice de qualidade do ajuste) e AGFI (Adjusted Goodness-of-fit Index) que são indicadores do ajuste ponderado, relacionam-se com proporção de variância-covariância nos dados explicada pelo modelo, com valores variando de 0 a 1 . O valor de parâmetro para aceitação deste é superior ou próximo ao intervalo de 0,90 a 0,95 ; e f) $\lambda$ (Coeficiente lambda), que é um indicador nominal do procedimento de regressão, seus resultados variam de 0 a 1 , quanto mais próximos de 1 , melhor o desempenho de predição da variável para o modelo.

Por fim, com a finalidade de compreender aspectos divergentes e convergentes do instrumento, realizaram-se procedimentos de correlação e ANOVA, tomando como base 
Tabela 3. Autovalores empíricos e aleatórios dos primeiros componentes da Escala de Atitudes e Crenças sobre Amor obtidos por meio da análise paralela

\begin{tabular}{|c|c|c|c|c|c|c|c|c|}
\hline \multirow[t]{2}{*}{ Autovalores } & \multicolumn{8}{|c|}{ Componentes } \\
\hline & 1 & 2 & 3 & 4 & 5 & 6 & 7 & 8 \\
\hline Empírico & 4,77 & 4,67 & 2,94 & 2,61 & 2,14 & 1,72 & 1,23 & 1,14 \\
\hline Aleatório & 1,49 & 1,44 & 1,40 & 1,37 & 1,34 & 1,31 & 1,29 & 1,26 \\
\hline
\end{tabular}

as dimensões resultantes da escala de estilos de amor, os aspectos multidimensionais da qualidade do relacionamento e variáveis demográficas.

\section{Procedimentos de coleta de dados e aspectos éticos}

Em conformidade com as normas do Conselho Nacional de Saúde, primeiramente foi analisada e aprovada a viabilidade do estudo via avaliação do Comitê de Ética em Pesquisa com Seres Humanos (CEPSH) da Universidade Federal de Santa Catarina. Com a aprovação, a coleta de dados foi realizada em locais públicos das cidades que compuseram a amostra. A aplicação ocorreu tanto de forma individual quanto coletivamente. Ao se constatar disponível o participante, o pesquisador abordava-o e realizava o convite de participação. A partir da aceitação, o questionário era entregue ao participante e, no processo de preenchimento, o pesquisador ficava à disposição para quaisquer dúvidas que tivessem. Concluído o processo, o termo de consentimento livre e esclarecido era entregue em duas cópias e assinado pelo participante, atendendo assim as normas vigentes.

\section{Resultados}

\section{Análise exploratória e indicadores de confiabilidade}

Procedeu-se primeiramente com uma análise dos componentes principais para verificação da fatorabilidade da matriz de dados. O KMO obteve o valor de 0,81 , e o teste de esfericidade de Bartlett foi significativo $(p<0,001)$. Para a decisão do número de fatores a serem extraídos, optou-se pelo método da análise paralela (Enzmann, 1997). A Tabela 3 apresenta os autovalores empíricos e os aleatórios da matriz comparativa.

Verifica-se que até o fator 6 , os autovalores empíricos são superiores aos aleatórios. A partir do componente 7 em diante, os valores empíricos são menores do que o valor aleatório, apontando para uma solução de 6 fatores, aspecto esperado a partir do modelo teórico de origem.

Definiu-se então a extração de seis fatores, assim como na versão anterior da medida e na versão original no idioma inglês (Hendrick \& Hendrick, 1986). Utilizou-se como método extração, a análise fatorial dos eixos principais (principal axis factoring). A rotação escolhida foi a promax, pelo fato de esta ser oblíqua e permitir correlação entre os fatores (Pasquali, 2005), aspecto justificado pela provável interdependência de alguns fatores e também pelo bom ajuste à matriz de dados. A disposição dos itens é demonstrada na Tabela 4.
A versão final da escala contou com um total de 37 itens, dispostos em uma estrutura fatorial de seis dimensões, conforme modelo teórico da versão original da medida brasileira (De Andrade \& Garcia, 2009). Desta forma, o primeiro fator, nomeado de "Agape", contemplou um total de sete itens, obtendo um coeficiente de confiabilidade alfa de Cronbach de 0,84, explicando $11,7 \%$ da variância. Essa dimensão avaliou aspectos ligados ao excesso de doação do companheiro de relacionamento para o outro membro da relação, envolvendo aspectos de sofrimento em prol do bem-estar do outro, cuidado e perda de sentido de vida diante de acontecimentos negativos com o companheiro(a).

O fator 2, chamado de estilo "Ludus", contemplou um total de cinco itens, obtendo um coeficiente de confiabilidade alfa de Cronbach de 0,82 e explicando 9,5\% da variância dos dados. Esse fator avalia aspectos ligados a um perfil individual de interação romântica menos comprometido com o relacionamento, caracterizando-se por comportamentos que indicam infidelidade, evitação de relações profundas, envolvendo também "jogos" de baixo comprometimento com os parceiros de relacionamento.

O fator 3, nomeado de "Eros", teve em sua versão final um total de sete itens, com um coeficiente de confiabilidade alfa de Cronbach de 0,75 e explicando 7,7 \% da variância dos dados. No conjunto, os itens dessa dimensão avaliam elementos ligados aos componentes eróticos, sensuais e sexuais de uma relação diádica, perpassando aspectos de atração, desejo e prazer.

O quarto fator foi o estilo "Pragma". Este obteve na sua estrutura final um coeficiente de confiabilidade de 0,69, avaliados por sete itens que explicaram no seu conjunto $6,6 \%$ da variância dos dados. A dimensão "Pragma” caracteriza o estilo de amor racional, marcado por comportamentos de indivíduos que operam num nível mais da razão do que da emoção, marcado por condutas de planejamento e busca de relacionamentos por aspectos de compatibilidade.

O quinto estilo foi o "Storge". A subescala ficou ao final estruturada com cinco itens, obtendo um coeficiente de confiabilidade alfa de Cronbach de 0,73. O fator avaliou um estilo romântico marcado por estereótipos de amizade e companheirismo na interação romântica, caracterizando pessoas que atribuem a seus parceiros de relacionamento representações de amizade.

Por fim, o sexto estilo foi o "Mania". Este contou com seis itens ao final e obteve um alfa de Cronbach de 0,65, explicando 4,5\% da variância dos dados. O estilo "Mania" caracteriza um perfil considerado imaturo e não saudável para interação conjugal, operacionalizado por comportamentos de ciúme, vigília e controle. 
Tabela 4. Matriz Fatorial Escala de Crenças sobre Amor - Rotação Promax

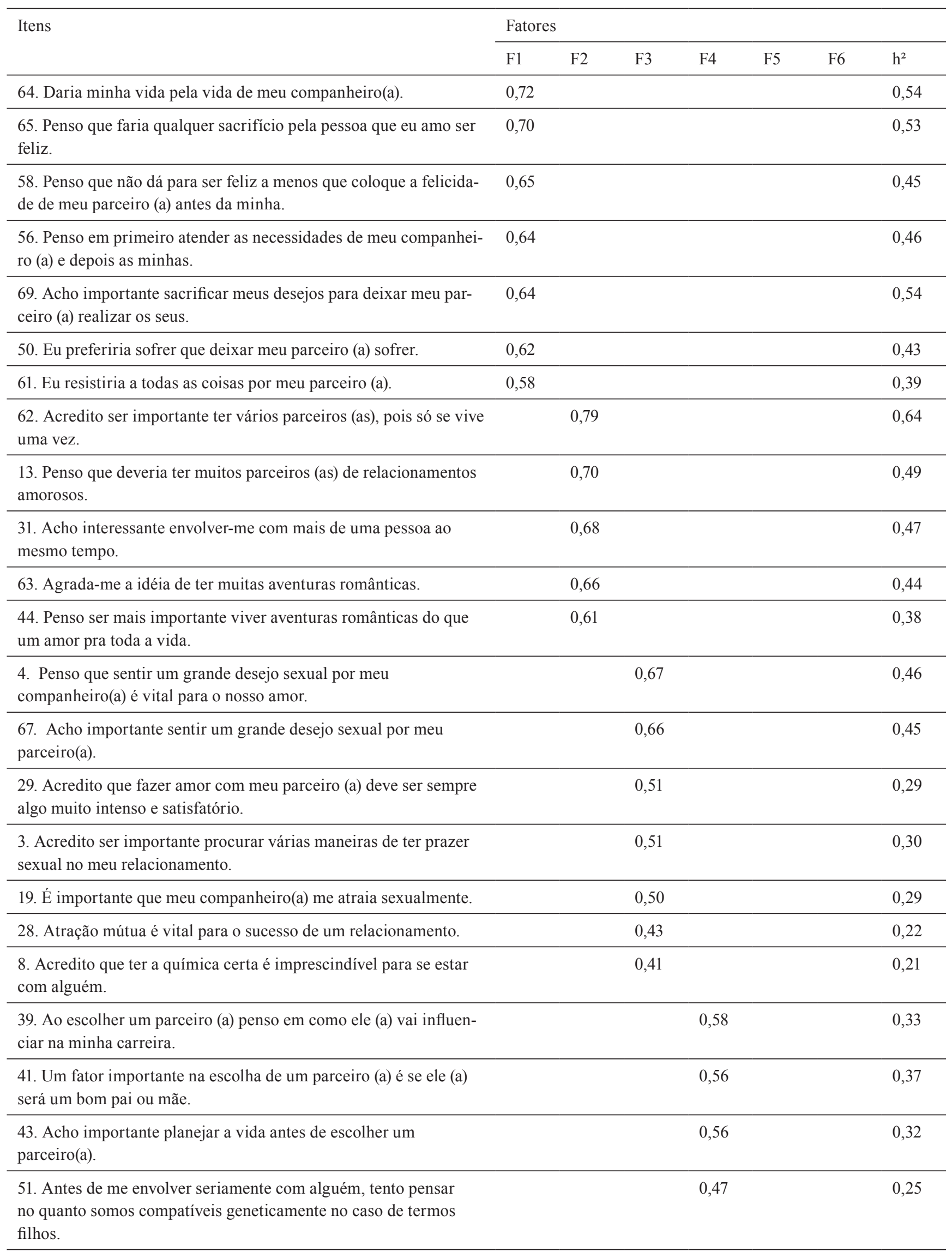


Continuação...

14. Acho importante considerar o que uma pessoa vai se tornar na vida antes de me comprometer com ela.

59. Uma consideração importante ao escolher um parceiro(a) é saber o que minha família acha dele.

33. Acho que para escolha de um companheiro (a) é preciso utilizar a razão.

0,46 0,26

26. Na minha opinião amor e amizade são muito parecidos.

17. Penso que o amor de verdade é uma amizade profunda.

48. Acredito que os melhores relacionamentos de amor se desenvolveram a partir de boas amizades

32. Penso que com o decorrer do tempo as relações amorosas ficam muito parecidas com amizades.

30. Penso que em meus romances a amizade transformou-se gradativamente em amor.

49. Penso ser importante vigiar as pessoas com que meu companheiro(a) anda.

\begin{tabular}{|c|c|c|c|c|c|c|c|}
\hline 47. Sinto ciúmes por tudo o que meu companheiro (a) faz. & & & & & & 0,63 & 0,42 \\
\hline $\begin{array}{l}\text { 36. Acho importante saber tudo que acontece na vida de meu } \\
\text { companheiro(a). }\end{array}$ & & & & & & 0,48 & 0,32 \\
\hline $\begin{array}{l}\text { 6. Acho difícil relaxar quando sei que meu parceiro (a) está com } \\
\text { outra pessoa. }\end{array}$ & & & & & & 0,36 & 0,14 \\
\hline 68. Acho que se fosse traído (a) cometeria uma loucura. & & & & & & 0,33 & 0,17 \\
\hline $\begin{array}{l}\text { 52. Penso que se meu parceiro (a) não presta atenção em mim } \\
\text { minha vida não tem graça. }\end{array}$ & & & & & & 0,30 & 0,18 \\
\hline Total de itens & 7 & 5 & 7 & 7 & 5 & 6 & \\
\hline Coeficiente de confiabilidade & 0,84 & 0,82 & 0,75 & 0,69 & 0,73 & 0,63 & \\
\hline Variância explicada por fator & 11,7 & 9,5 & 7,7 & 6,6 & 5,3 & 4,5 & 45,3 \\
\hline
\end{tabular}

\section{Análise confirmatória e indicadores de confiabilidade}

A análise fatorial confirmatória da escala de estilos de amor foi realizada tomando como base a matriz fatorial apresentada na Tabela 4. O conjunto de itens dispostos no modelo de seis dimensões foram inicialmente testados sem inter-relação dos fatores. Os resultados de ajuste desse primeiro modelo não foram plenamente satisfatórios $[\chi 2=2019,745$, g.l. $=629(p<0,0001), \chi 2 / \mathrm{gl}=3,211, \mathrm{RMR}=0,102, \mathrm{GFI}=$ $0,87, \mathrm{AGFI}=0,85, \mathrm{CFI}=0,81, \mathrm{RMSEA}(90 \% \mathrm{CI})=0,54(0,51$ - 0,57]. Buscando aprimorar os resultados, observaram-se os índices de modificação do modelo, os quais sugeriram o estabelecimento inter-relações entre as variáveis latentes de estilos de amor. Com as devidas modificações, obtiveram-se os seguintes indicadores de ajuste: $[\chi 2=1766,310$, g.1. $=614$ $(p<0,0001), \chi 2 / \mathrm{gl}=2,877, \mathrm{RMR}=0,066, \mathrm{GFI}=0,88, \mathrm{AGFI}$ $=0,87 . \mathrm{CFI}=0,85$, RMSEA $(90 \% \mathrm{CI})=0,50(0,47-0,52]$. Apesar de o modelo resultante ser melhor que o anterior, decidiu-se novamente pela verificação dos índices de modificação e se encontraram valores elevados entre os pares de parâmetros de erros de alguns itens (e17-e26 = 19,57; e13 - e $31=27,53$; e65 - e64 = 46,98; e3 - e4 = 19,57). Para contornar o não ajuste adequado dos dados, estabeleceu-se uma covariância entre erros dos itens indicados. O modelo resultante apresentou indicadores mais adequados que os outros dois anteriores: $[\chi 2=1601,190$, g.1. $=610(p<0,0001)$, $\chi 2 / \mathrm{gl}=2,625, \mathrm{RMR}=0,065, \mathrm{GFI}=0,89, \mathrm{AGFI}=0,88$. CFI $=0,87$, RMSEA $(90 \% \mathrm{CI})=0,46(0,44-0,49]$.

Para finalizar a exposição dos indicadores da medida na segunda parte do banco de dados, calcularam-se os coeficientes de consistência interna (alfa de Cronbach): estilo Agape $(0,84)$, estilo Ludus $(0,82)$, estilo Eros $(0,80)$, estilo Pragma $(0,71)$, estilo Mania $(0,68)$ e estilo Storge $(0,67)$, todos eles no seu conjunto geral apresentando resultados aceitáveis (Nunnally, 1978). 


\section{Estilos de amor e construtos associados}

Com objetivo de verificar aspectos convergentes e divergentes da medida resultante, procedeu-se a um conjunto de análises de diferenças entre variáveis critérios (sexo e término de relacionamento) e de correlação (aspectos multidimensionais da qualidade do relacionamento). Nas análises apresentadas na sequência, tomou-se como base o número de participantes que responderam de forma completa o conjunto de instrumentos da pesquisa $(\mathrm{N}=1322)$.

As diferenças entre sexo a partir da ANOVA foram significativas para os estilos Ludus $(\mathrm{F}=103,125 ; \mathrm{p}=0,000)$, Eros $(\mathrm{F}=15,252 ; \mathrm{p}<0,001)$ e Agape $(\mathrm{F}=88,262 ; \mathrm{p}<0,001)$. Nos estilos Mania $(F=1,471 ; p=0,225)$, Pragma $(F=0,063 ; p$ $=0,802)$ e Storge $(F=0,950 ; p=0,330)$ não se observaram diferenças entre os sexos. Nos três estilos com diferenças significativas de média entre homens e mulheres, foram verificados escores mais elevados entre os participantes do sexo masculino (Ludus, $\mathrm{m}=2,20$; Eros $\mathrm{m}=3,71$; Agape $\mathrm{m}$ $=2,65$ ) do que feminino (Ludus, $\mathrm{m}=1,81$; Eros $\mathrm{m}=3,58$; Agape $\mathrm{m}=2,27$ ).

No que diz respeito às diferenças no estilos de amor entre os participantes que permaneciam num relacionamento romântico e aqueles que não permaneciam, observou-se que os participantes que do grupo dos que terminaram possuíam escores mais elevados em na característica do tipo Agape $(\mathrm{F}=$ $14,837 ; \mathrm{p}<0,001)$ e Ludus $(\mathrm{F}=87,543 ; \mathrm{p}<0,001)$. No estilo Agape, os participantes que permaneciam em relacionamento $(\mathrm{m}=2,50)$ obtiveram escores mais elevados do que aqueles que terminaram $(\mathrm{m}=2,30)$. Já no estilo Ludus, a situação foi contrária, indivíduos que terminaram seu relacionamento (m $=2,26$ ) tiveram escores mais elevados do que aqueles que não terminaram $(\mathrm{m}=1,86)$.

Por fim, para verificar a relação dos construtos tipológicos do amor com aspectos da qualidade no relacionamento romântico, estabeleceram-se correlações entre as dimensões da escala de estilos de amor com as variáveis multidimensionais da qualidade do relacionamento (sexo, comunicação, comprometimento e compromisso) mensurados pela Aquarela-R (De Andrade \& Garcia, 2012). A Tabela 5 apresenta os resultados da respectiva análise.
Conforme Tabela 5, observam-se correlações significativas e positivas do estilo Mania com aspectos de qualidade sexual $(0,11)$ e intimidade $(0,07)$; do estilo Eros com aspectos qualidade sexual $(0,28)$ e intimidade $(0,17)$. No estilo Agape, correlações entre qualidade da intimidade $(0,11)$, da comunicação $(0,15)$ e de comprometimento $(0,22)$. Quanto à relação negativa das variáveis, observou-se no estilo Ludus correlações negativas entre qualidade sexual $(-0,06)$, intimidade $(-0,25)$, comunicação $(-0,27)$ e compromisso $(-0,35)$, e por fim, o estilo Storge com aspectos de qualidade sexual $(-0,08)$.

\section{Discussão}

$\mathrm{O}$ aspecto a se destacar inicialmente sobre o presente estudo diz respeito ao fato de contarmos ao final com um instrumento psicometricamente validado para investigação de aspectos da esfera cognitiva e atitudinal do indivíduo nos seus relacionamentos românticos dentro do contexto sócio-cultural brasileiro. A escala de estilos de amor aqui apresentada obteve dados mais satisfatórios e adequados do que os produzidos na versão anterior do instrumento (De Andrade \& Garcia, 2009), fato este atribuído ao aprimoramento e aumento no número de itens originais da medida, possibilitando dessa forma uma estruturação de um instrumento com representantes comportamentais mais precisos e menos ambíguos.

Os coeficientes de confiabilidade da medida foram mais elevados que na versão anterior, e se mantiveram estáveis quando comparados com a amostra da CFA, sendo estes também mais elevados que a versão apresentada a partir da tradução da versão portuguesa por Cassepp-Borges (2010).

Quanto aos resultados da análise fatorial confirmatória, os resultados do ajuste aproximaram-se do que se espera (Byrne, 2010). Dos diversos índices tomados como referência, três indicadores ficaram pouco abaixo da faixa desejada (CFI, GFI e AGFI), todavia muito próximos do esperado para excelência do modelo. Das modificações realizadas no ajuste, tomadas com base na orientação dos índices de modificação, é importante destacar que os dois modelos de melhor ajuste preveem a relação estabelecida entre todos

Tabela 5. Matriz de correlações da escala de estilo de amor e dimensões da qualidade do relacionamento romântico.

\begin{tabular}{|c|c|c|c|c|}
\hline \multirow{2}{*}{ Sub-escalas Crenças } & \multicolumn{4}{|c|}{$\begin{array}{c}\text { Dimensões de } \\
\text { Qualidade no Relacionamento }\end{array}$} \\
\hline & Q.Sex & Q.Int. & Q.Com. & Q.Comp. \\
\hline Mania & $0,11^{* *}$ & $0,07^{*}$ & 0,00 & 0,04 \\
\hline Ludus & $-0,06^{*}$ & $-0,25^{* *}$ & $-0,27 * *$ & $-0,35^{* *}$ \\
\hline Eros & $0,28^{* *}$ & $0,17 * *$ & $-0,03$ & $-0,01$ \\
\hline Pragma & $-0,02$ & $-0,05$ & $-0,04$ & $-0,02$ \\
\hline Storge & $-0,08^{* *}$ & 0,03 & 0,02 & 0,02 \\
\hline Agape & 0,05 & $0,11^{* *}$ & $0,15^{* *}$ & $0,22 * *$ \\
\hline
\end{tabular}

Legenda: Q.Sex (qualidade sexual); Q.Int. (qualidade da intimidade); Q.Com. (qualidade da comunicação) e Q.Comp (qualidade do compromisso).

$*<0,05 * *<0,001$ 
os fatores latentes dos estilos de amor. O presente resultado de certa forma associa-se à premissa de interpendência entre os fatores, apontada na versão original de Hendrick e Hendrick (1986) no estudo com amostra de portugueses de Neto (2002) e no estudo transcultural em Portugal, Angola, Moçambique, Cabo Verde, Brasil, França, Suíça (Neto et al. 2000), no entanto não deixa clara, conforme também sinaliza Cassepp-Borges (2010), a relação de fatores de primeira e segunda ordem no modelo.

Quanto aos aspectos de convergência e divergência dos seis construtos dos estilos de amor com a variável sexo, permanência no relacionamento e qualidade, os resultados orientam conclusões discriminantes da medida adaptada ao contexto brasileiro, assim como a convergência com outros aspectos já levantados em estudos com outras populações. Quanto ao sexo dos participantes, as pontuações mais elevadas no estilo Eros demonstram a percepção mais voltada dos homens para aspectos da natureza sexual e excitante do relacionamento, resultado divergente do estudo original de Hendrick e Hendrick (1986) e também do estudo transcultural de Neto et al. (2000), no qual homens e mulheres tinham pontuações semelhantes. No que diz respeito ao nível mais elevado estilo Ludus em homens, resultados semelhantes foram referenciados no estudo original de Hendrick e Hendrick (1986) com amostra de americanos. Nesse aspecto, homens possuem atitudes mais permissivas, mulheres por sua vez, são mais conservadoras. Quanto ao estilo Agape, o maior nível na amostra de homens revela uma atitude de maior doação dos participantes deste sexo. Nenhuma consideração em outros artigos foi encontrada para justificar tal resultado, deixando a pergunta em aberto para investigações futuras.

Quanto aos aspectos do término ou não do relacionamento, destaca-se a dificuldade de manutenção de relações estáveis em participantes com níveis mais elevados de Ludus. Tanto os dados a partir de diferença entre os grupos de participantes que terminaram e os que não terminaram, quanto às correlações negativas com aspectos multidimensionais do relacionamento, demonstram que atitudes aventureiras num relacionamento romântico sinalizam aspectos de perda da qualidade e término. Jonason e Kavanagh (2010) sinalizam o estilo Ludus como uma característica limitante do sistema emocional da empatia, um jogo afetivo lúdico favorece o distanciamento de uma ligação afetiva saudável.

A correlação negativa baixa, mas existente entre estilo Storge e qualidade sexual, demonstra num certo nível, o mecanismo de perda de desejo ou "química" entre as características românticas do traço Storge, marcado por atribuições de amizade ao companheiro (a) romântica, e os aspectos mais sensuais e sexuais do relacionamento. Aspecto parecido com este é mencionado em trabalho clássico Hatfield, Traupmann, e Walster (1978) com amostra de americanos, e Sáchez Aragón (2005) num estudo com mexicanos, nos quais são sinalizadas distinções entre amor passional e amor companheiro. No primeiro, a díade volta-se para uma dimensão mais sexual do relacionamento, comum em relações de curta duração. No segundo por sua vez, os integrantes do relacionamento compartilham maiores níveis de intimidade e apoio mútuo, algo bastante característico do estilo Storge.
Quanto ao estilo Eros, as correlações entre qualidade sexual esse perfil. Dimensões de paixão são comumente preditoras da qualidade sexual (Ratelle, Carbonneau, Vallerand, \& Mageau, 2013).

Como indicação para futuros estudos relativos à medida de estilos de amor, sugere-se o aprimoramento das subescalas com número de itens reduzido e coeficientes de confiabilidade abaixo de 0,70 , sendo o caso das subescalas de estilo controle e estilo racional. $\mathrm{O}$ acréscimo de itens relativos a essas dimensões pode aprimorar a confiabilidade da medida. Na perspectiva teórica dos estilos de amor, são sugeridos estudos com outras variáveis do contexto dos relacionamentos, como por exemplo: estilos de apego, componentes do amor e duração do relacionamento.

Entender o amor e seus determinantes a partir de modelos matemáticos e instrumental quantitativo não é uma tarefa fácil, e nem tão pouco conclusiva. No Brasil, esse movimento dentro de uma perspectiva acadêmica ainda é recente, e se espera que com as contribuições desta pesquisa e de outros autores (Scorsolini-Comin \& Santos, 2010), ocorra um maior destaque e investimento no assunto por parte daqueles que trabalham ou estudam elementos da vida de um casal.

Os aspectos descritos possuem no seu conjunto, além do conhecimento, a tarefa de orientar futuras pesquisas envolvendo aspectos dos relacionamentos românticos e também atividades práticas ligadas ao campo da psicoterapia de família e casal, trazendo desta forma uma orientação de caráter mais científico para que aqueles que atuam na área.

\section{Referências}

Alferes, V. R. (1996). Atração interpessoal, sexualidade e relações íntimas. In J. Vala (Ed.), Psicologia social. (pp. 113-139). Lisboa: Fundação Calouste Gulbenkian.

Bersheid, E., \& Walster, E. (1969). Interpersonal Attraction. Reading, MA: Addison Wesley.

Byrne, B. M. (2010). Structural Equation Modeling with Amos: basic concepts, applications, and programming. New York: Routledge.

Cassep-Borges, V. (2010). Amor e construtos relacionados: evidencias de validade de instrumentos de medida no Brasil. Tese de doutorado não publicada, Universidade de Brasília, Brasília.

Colins, N. L., \& Read, S. J. (1990). Adult attachment, workind models, and relationship quality in dating couples. Journal of Personality and Social Psychology, 58, 644-663.

Contreras, R., Hendrick, S. S., \& Hendrick, C. (1996). Perspectives on marital love and satisfaction in Mexican American and Anglo-American couples. Journal of Counseling and Development, 74, 408-415.

De Andrade, A. L., \& Garcia, A. (2012). Desenvolvimento de uma medida multidimensional para avaliação de qualidade em relacionamentos românticos - Aquarela-R. Psicologia: Reflexão e Crítica, 25(4), 634-643. Retrieved from http:// dx.doi http://dx.doi.org/10.1590/S0102-79722012000400002

De Andrade, A. L., \& Garcia, A (2009). Atitudes e crenças sobre o amor: versão brasileira da Escala de Estilos de Amor. Interpersona, 3(1), 89-102. 
Enzmann, D. (1997). RanEigen: A program to determine the parallel analysis criterion for the number of principal components. Applied Psychological Measurement, 21, 232.

Ferrer Pérez, V. A., Bosch Fiol, E., Navarro Guzmán, C., Ramis Palmer, M. C., \& García Buades, E. (2008). El concepto de amor en España. Psicothema, 20(4), 589-595.

Hair, J. F. Jr., Anderson. R. E., Tatham, R. L., \& Black, W. C. (2005). Análise Multivariada de Dados. Porto Alegre: Bookman.

Hatfield, E., Traupmann, J., \& Walster, G. W. (1978). Equity and extramarital sexuality. Archives of Sexual Behavior, 7, 127-141.

Hazan, C., \& Shaver, P. R. (1987). Romantic love conceptualized as an attachment process. Journal of Personality and Social Psychology, 52, 511-524.

Hendrick, C., \& Hendrick, S. S. (1986). A theory and method of love. Journal of Personality and Social Psychology, 50, 392-402.

Hendrick, C., \& Hendrick, S. S. (2006). Styles of Romantic Love. In R. J. Sternberg, \& K. Weis (Eds.), The New Psychology of Love. (pp. 149-170). Yale University.

Hendrick, C., Hendrick, S. S., \& Adler, N. L. (1988). Romantic relationship: Love, satisfaction, and staying together. Journal of Personality and Social Psychology, 54, 980-988.

Hendrick, C., Hendrick, S. S., \& Dicke, A. (1998). The love attitudes scale: short form. Journal of Social and Personal Relationships, $15,147-159$

Jonason, P. K., \& Kavanagh, P. (2010). The dark side of love: The Dark Triad and love styles. Personality and Individual Differences, 49(6), 606-610.

Kanemasa, Y., Taniguchi, J., \& Daibo, I. (2004). Love styles and romantic love experiences. Social Behavior and Personality, $32,265-282$.

Lee, J. A. (1977). A typology of styles of loving. Personality and Social Psychology Bulletin, 3(2), 173-182.

Masuda, M. (2003). Meta-analyses of love scales: do various love scales measure the same psychological constructs? Japanese Psychological Research, 45(1), 25-37.

Neto, F. (2002). Colors associated with styles of love. Perceptual and Motor Skills, 94, 1303-1310.
Neto, F., Mullet, E., Deschamps, J. C., Barros, J., Benvindo, R. Camino, L., ... Machado, M. (2000). Cross-Cultural variations in attitudes toward love. Journal of Cross-Cultural Psychology, 31(5), 626-635.

Nunnally, J. C. (1978). Psychometric theory. New York: McGrawHill.

Pasquali, L. (2005). Rotação dos Fatores. In L. Pasquali (Org.), Análise Fatorial para Pesquisadores. (pp. 87-103). Brasília: LabPam.

Ratelle., C. Carbonneau, N. Vallerand, R., \& Mageau, G. (2013). Passion in the romantic sphere: A look at relational outcomes. Motivation and Emotion, 37(1), 106-120.

Sánchez Aragón, R. (2005). Mexican Love Styles. In A. Garcia (Ed.), Personal Relationships: International Studies. (64-77). Vitória - ES: GM Gráfica e Editora

Scorsolini-Comin, F., \& dos Santos, M. (2010). Satisfação Conjugal: Revisão Integrativa da Literatura Científica Nacional. Psicologia: Teoria e Pesquisa, 26(3), 525-532.

Sternberg, R. J. (1986). A triangular theory of love. Psychological Review, 93, 119-135.

Vohs, K. D., Finkenauer, C., \& Baumeister, R. F. (2010). The sum of friends' and lovers' self-control scores predicts relationship quality. Social Psychological and Personality Science, 2(2), 138145. Retrieved from http://dx.doi:10.1177/1948550610385710

White, J. K., Hendrick, S. S., \& Hendrick, C. (2004). Big five personality variables and relationship constructs. Personality an Individual Differences, 37, 1519-1530.

Recebido em 01.03.2011

Primeira decisão editorial em 19.08.2013

Versão final em 23.10.2013

Aceito em 06.11.2013 